\title{
Pathophysiology of Schizophrenia Based on Impaired Glial-Neuronal Interactions
}

\author{
Bernhard J. Mitterauer \\ Volitronics-Institute for Basic Research, Psychopathology and Brain Philosophy, \\ Gotthard Guenther Archives, Wals/Salzburg, Austria \\ Email: mitterauer@wasi.tv
}

Received November 5, 2013; revised December 5, 2013; accepted December 15, 2013

Copyright (c) 2014 Bernhard J. Mitterauer. This is an open access article distributed under the Creative Commons Attribution License, which permits unrestricted use, distribution, and reproduction in any medium, provided the original work is properly cited. In accordance of the Creative Commons Attribution License all Copyrights (c) 2014 are reserved for SCIRP and the owner of the intellectual property Bernhard J. Mitterauer. All Copyright (C) 2014 are guarded by law and by SCIRP as a guardian.

\begin{abstract}
The model of impaired glial-neuronal interactions in schizophrenia is based on the core hypothesis that nonfunctional astrocyte receptors may cause an unconstrained synaptic information flux such that glia lose their modulatory function in tripartite synapses. This may lead to a generalization of information processing in the neuronal networks responsible for delusions and hallucinations on the behavioral level. In this acute paranoid stage of schizophrenia, non-functional astrocytic receptors or their loss decompose the astrocyte domain organization with the effect that a gap between the neuronal and the glial networks arises. If the illness progresses the permanent synaptic neurotransmitter flux may additionally impair the oligodendrocyte-axonic interactions, accompanied by a "creeping" decay of oligodendroglia, axons and glial gap junctions responsible for severe cognitive impairment. Here we may deal with after-effects caused by the basic fault of information processing in tripartite synapses. The gaps between the neuronal and glial networks prohibit the neuronal reality testing of intentional programs presumably generated in the glial networks, called schizophrenic dysintentionality. In non-schizophrenic delusions glia may not be disturbed, but exhausted extrasynaptic information processing may cause an unconstrained synaptic flux responsible for delusions.
\end{abstract}

\section{KEYWORDS}

Schizophrenia; Glial-Neuronal Interactions; Impairments

\section{Introduction}

The brain is basically equipped with two cell systems, the neuronal and the glial cell system. Meanwhile, the modifying function of glia in their interaction with neurons is experimentally verified. This concerns both physiology and pathophysiology [1]. Therefore, it seems necessary to search for impairments in glial-neuronal interactions that may be responsible for the pathophysiology of schizophrenia. Meltzer [2] challenged psychiatric research by posing the question how long schizophrenia will exist as an entity and what will be its future name or names, since this is diffcult to predict. Here, I propose a pathophysiological model of schizophrenia that attempts to show that Bleuler's term may be appropriate. Disconnections in the neuronal networks in and between areas of brains with schizophrenia are experimentally well established [3]. The underlying disconnection hypothesis states that schizophrenia can be understood both in cognitive and pathophysiological terms as a failure of proper functional integration within the brain [4]. Accordingly, schizophrenia may be best understood in terms of abnormal interactions between different brain regions [5, $6]$.

However, structural, molecular and functional changes in glial cells (astrocytes, oligodendrocytes and microglial cells) have become a major focus of interest in search for the pathophysiological foundations of schizophrenia. Many studies show abnormalities in the connecting elements between the nerve cell bodies (synapses, dendrites and axons) and in all three types of glial cells [7]. The findings of white matter abnormalities [8,9] suggest a focus on glial-neuronal interactions in schizophrenia re- 
search. Moreover, a deeper insight into the etiopathophysiology of this severe disorder is dependent on the underlying brain model from which the abnormalities are deduced. Schizophrenia is a complex illness that requires a reappraisal of exclusively neuronal models to include an astrocytic hypothesis of dysfunction which could lead to a better understanding of schizophrenia on the behavioral level and could even provide novel treatment strategies [10].

\section{Hypotheses}

My core hypothesis is this: astrocytes are equipped with receptors for the various neurotransmitter substances. The occupancy of these receptors by neurotransmitters activates the release of gliotransmitters from the astrocyte modulating neuronal synaptic transmission. This may basically occur by occupying cognate receptors for gliotransmitters on the presynapse such that the synaptic information processing is temporarily turned off. If the receptors on astrocytes are non-functional or cannot even be expressed, an unconstrained synaptic flux arises. Since each astrocyte via its processes contacts a distinct amount of synapses generating a glial-neuronal domain, in the case of non-functional astrocytic receptors the domain organization decomposes. Hence, the basic pathophysiological fault of schizophrenia may be caused by synaptic gaps between the neuronal components and the astrocyte.

In the acute delusional stage of the illness no significant network impairments may occur, but the functional separation between the neuronal and glial networks on the synaptic level may lead to uncontrolled synaptic information processing. Since the astrocyte domain organization is interrupted, the information processing generalizes in both networks which may be responsible for delusional misinterpretations of a given reality in the environment. In parallel, the unconstrained synaptic transmitter flux may impair oligodendrocyte-axonic interactions leading to the decay of both oligodendroglia and axons which may explain the cognitive and emotional defects of schizophrenics in the progressive stage of the illness. Supposing that in the glial networks intentional programs may be generated which are tested in the neuronal networks regarding their feasibility in the environment, a brain with schizophrenia is unable to do so because of the synaptic separation between the neuronal and glial networks. One can speak of schizophrenic dysintentionality.

What non-schizophrenic delusions concern, these may be based on a different pathophysiology. Astrocytes also exert a modulating function in extrasynaptic neurotransmission via the activation of inhibitory interneurons that negatively feed back to the presynapse. If, for various reasons (e.g. stress), a synaptic neurotransmitter flooding occurs, although the astrocytic receptors are normally expressed an unconstrained information flux as in schizophrenic delusions may occur, but these are caused by a disturbance of the extrasynaptic negative feedback mechanism. Before further elaborating on these hypotheses, the underlying brain model will be outlined.

\section{Brain Model of Glial-Neuronal Interactions}

\subsection{Description of the Networks}

The proposed biological brain model is based on glialneuronal interactions $[11,12]$. The nervous tissue of the brain consists of the neuronal system (neurons, axons, dendrites) and the glial system (astrocytes, oligodendrocytes with myelin sheaths enfolding axons, radial glia, and microglia). Experimental results are inspiring a major reexamination of the role of glia in the regulation of neural integration in the central nervous system [13].

Figure 1 shows a schematic diagram of the glial-neuronal interaction: two astrocytes $\left(\mathrm{Ac}_{1,2}\right)$ are shown in this very simple model, whereby in each case only one neuron $\left(\mathrm{N}_{1,2}\right)$ belonging to an astrocyte is taken into consideration. [14] identified how a single astrocyte contacts only four to eight neurons, but 300 to 600 synapses via its processes. The glial network (syncytium) consists in this schema of two astrocytes and two oligodendrocytes $\left(\mathrm{Oc}_{1,2}\right)$ interconnected via gap junctions (g.j.). The neuronal system shows two neurons $\left(\mathrm{N}_{1,2}\right)$ with two afferent axons $\left(\mathrm{Ax}_{\mathrm{i}, \mathrm{j}}\right)$ and two afferent axodendritic synapses $\left(\mathrm{Sa}_{\mathrm{i}, \mathrm{j}}\right)$, two efferent axons $\left(\mathrm{Ax}_{1,2}\right)$ with myelin sheaths (Ms) and a node of Ranvier (N.R.), as well as two dendro-dendritic synapses $\left(\mathrm{Sd}_{1,2,3,4}\right)$ with the corresponding dendrites $\left(\mathrm{D}_{1} \mathrm{D}_{2} ; \mathrm{D}_{3} \mathrm{D}_{4}\right)$. In addition, a decrease of calcium $\left(\mathrm{Ca}^{2+}\right)$ in the extrasynaptic space (exsyspace) activates astrocytes that negatively feed back to the synapse [15].

\subsection{Outline of an Astrocytic Syncytium}

Different connexins allow communication between diverse cell populations or segregation of cells into isolated compartments according to their pattern of connexin expression. Gap junctions are composed of hemichannels (connexons) that dock to each other via their extracytoplasmic extremities. Each hemichannel is an oligomer of six connexin proteins (Cx). In the central nervous system, cell-specific and developmentally regulated expression of eight connexins has been demonstrated [16].

My hypothesis focuses on gap junctions between astrocytes, the main glial cell type besides oligodendrocytes and microglia. Gap junctions are considered to provide a structural link by which single cells are coupled to build a functional syncytium with a communication behavior that cannot be exerted by individual cells. 


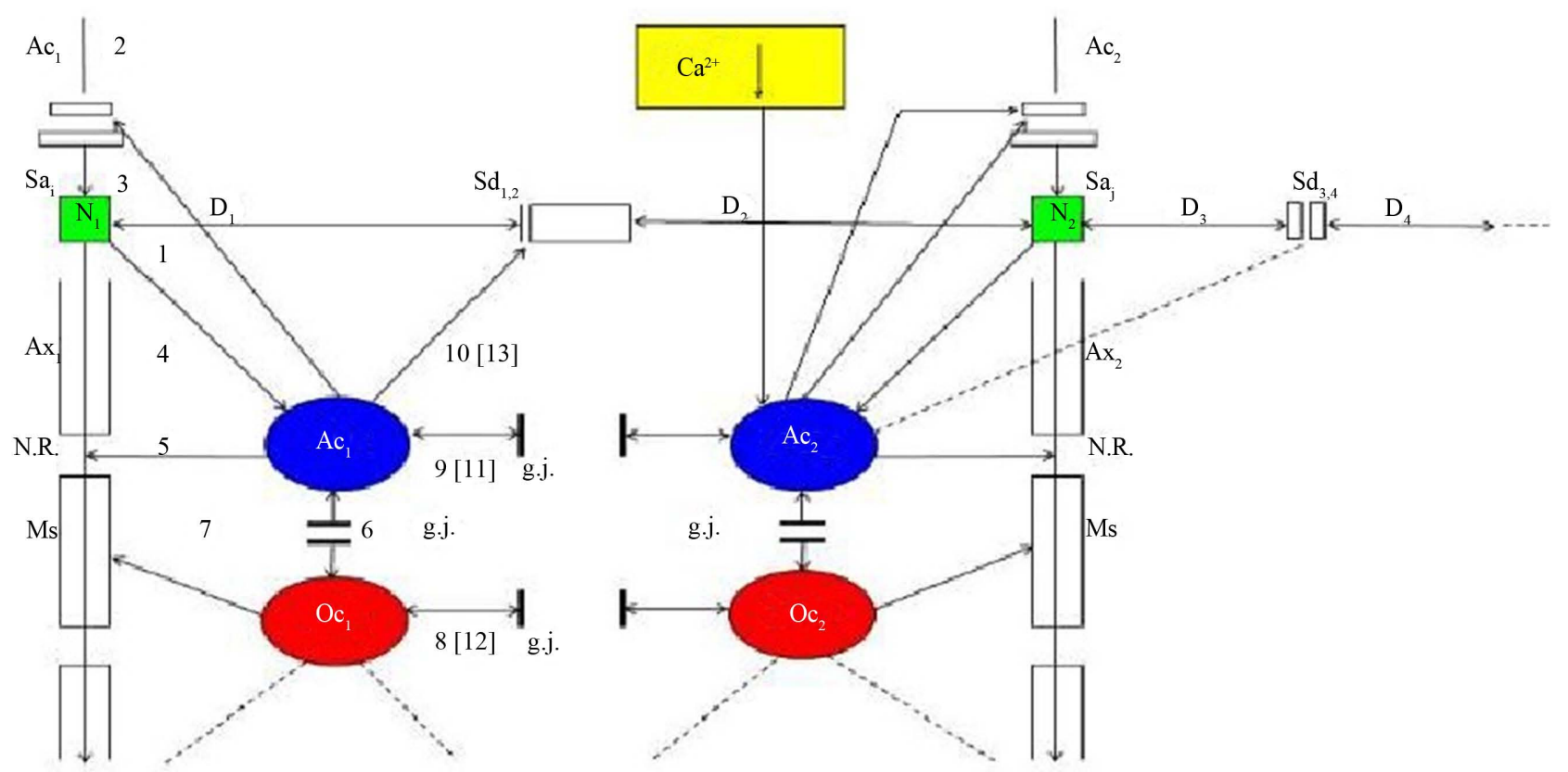

Figure 1. Schematic diagram of the glial-neuronal interaction. Two astrocytes $\left(\mathbf{A c}_{1,2}\right)$ are shown in this very simple model, whereby in each case only one neuron belonging to an astrocyte is taken into consideration. The glial network (syncytium) consists of two astrocytes and two oligodendrocytes $\left(\mathrm{Oc}_{1,2}\right)$ belonging to them. Gap junctions (g.j.) exist between the astrocytes and the oligodendrocytes. The neuronal system shows two neurons $\left(N_{1,2}\right)$ with two afferent axons $\left(A x_{i, j}\right)$ and two afferent axo-dendritic synapses $\left(\mathrm{Sa}_{\mathrm{i}, \mathrm{j}}\right)$, two efferent axons $\left(\mathbf{A x}_{1,2}\right)$ with myelin sheaths $(\mathrm{Ms})$ and a node of Ranvier $(\mathrm{N} . R$.$) , as well$ as two dendro-dendritic synapses $\left(\operatorname{Sd}_{1,2,3,4}\right)$ with the corresponding dendrites $\left(D_{1}, D_{2}, D_{3}, D_{4}\right)$. A decrease of calcium $\left(C a^{2+}\right)$ in the extrasynaptic space (ex sy space) activates astrocytes that negatively feed back to the synapse [15].

Gap junctions of an astrocytic syncytium consist of the four identified connexins Cx43, Cx32, Cx26 and Cx45, forming homotypic (i.e. gap junction channels formed by hemichannels of the same kind) and heterotypic gap junction channels (i.e. formed by hemichannels of different kinds). Whereas astrocytes are interconnected with their neighbors via gap junctions, the interactions of astrocytes with neurons occur mainly in synapses called tripartite synapses [17].

Figure 2 shows a diagrammatic scheme depicting an astrocytic syncytium. Six astrocytes $\left(\mathrm{Ac}_{1} \ldots \mathrm{Ac}_{6}\right)$ are completely interconnected via fifteen gap junctions (g.j.) according to the formula $\mathrm{n}: 2(\mathrm{n}-1)$. Each astrocyte contacts a neuronal synapse, building a tripartite synapse in the sense of a glial-neuronal unit. Admittedly, this simple diagram refers only to the elementary components and their connections in an astrocytic syncytium.

Since in the brain each macroscopic gap junction is an aggregate of many, often hundreds of tightly packed gap junction channels are observed [18]. The number and composition of gap junctions can be dynamically regulated at the level of the endoplasmic reticulum by either upregulating connexin biosynthesis or decreasing the rate of connexin degradation, and at the cell surface by enhancing gap junction assembly or reducing connexin degradation.

\subsection{Model of a Glutamatergic Tripartite Synapse}

The close morphological relations between astrocytes and synapses as well as the functional expression of relevant receptors in the astroglial cells prompted the appearance of a new concept known as the tripartite synapse, and which I call glial-neuronal synaptic unit (GNU). Araque et al. [17] showed that glia respond to neuronal activity with an elevation of their internal $\mathrm{Ca}^{2+}$ concentration which triggers the release of chemical transmitters from glia themselves, and, in turn, causes feedback regulation of neuronal activity and synaptic strength. Although a true understanding of how the astrocyte interacts with neurons is still missing, several models have been published [14]. Here, I focus on a modified model proposed by Newman [19].

Figure 3 represents the interaction of the main components of synaptic information processing as follows: sensori-motoric networks compute environmental information activating the presynapse (1). The activated presynapse releases glutamate (GL) from vesicles (v) that occupy both postsynaptic receptors (poR) and receptors on the astrocyte (acR) (2) (For the sake of clarity, only one receptor is shown). Moreover, glutamate may also activate gap junctions (g.j.) in the astrocytic syncytium leading to an enhanced spreading of $\mathrm{Ca}^{2+}$ waves (3). In 


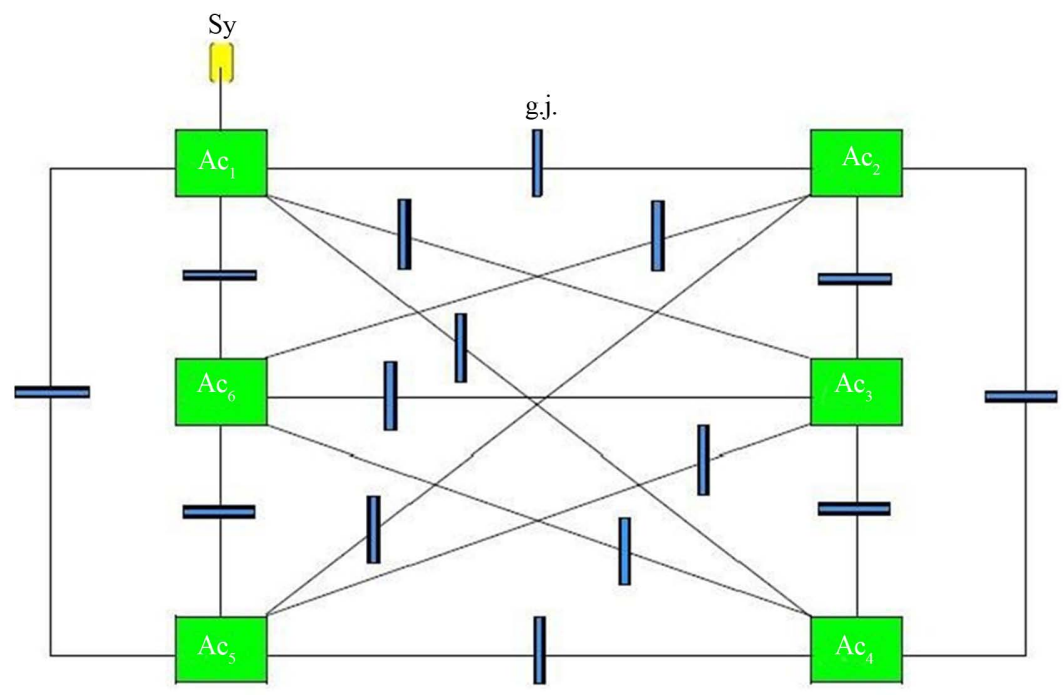

Figure 2. Outiline of an astrocytic syncytium. Six astrocytes $\left(\mathrm{Ac}_{1} \ldots \mathrm{Ac}_{6}\right)$ are interconnected via 16 gap junctions (g.j.) building a complete syncytium. Each astrocyte contacts a neuronal synapse representing a tripartite synapse (for the sake of clarity, only one synaptic contact [Sy] is shown).

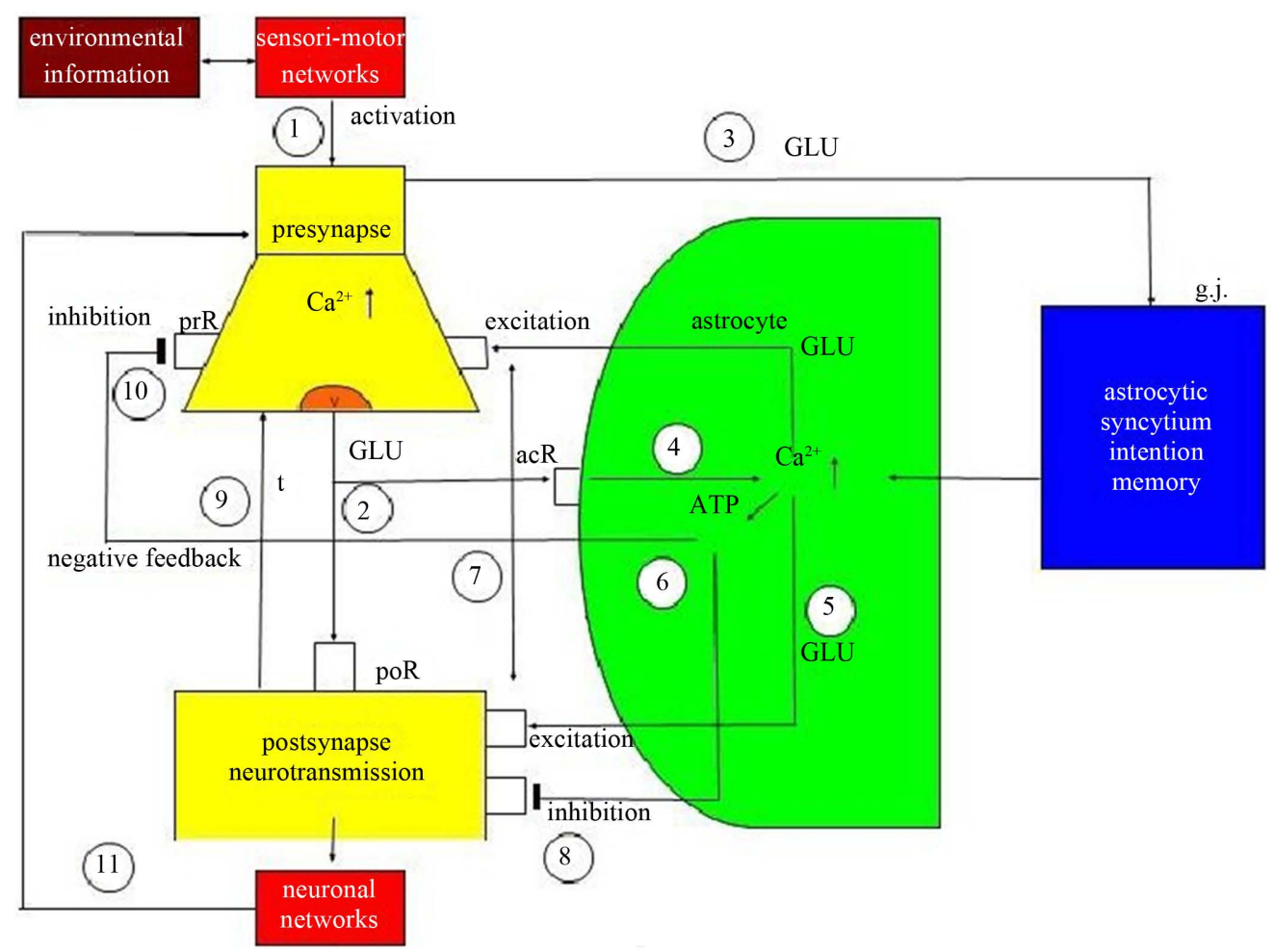

Figure 3. Schematic diagram of possible glial-neuronal interactions at the glutamatergic tripartite synapse (modified after Newman 2005). Sensori-motor networks compute environmental information activating the presynapse (1). The activated presynapse releases glutamate (GLU) from vesicles (v) occupying both postsynaptic receptors (poR) and receptors on the astrocyte (acR) (2). GLU also activates gap junctions (g.j.) in the astrocytic syncytium, enhancing the spreading of $\mathrm{Ca}^{2+}$ waves (3). In parallel, the occupancy of acR by GLU also activates $\mathrm{Ca}^{2+}$ within the astrocyte (4). This mechanism exerts the production of GLU (5) and adenosinetriphosphate (ATP) (6) within the astrocyte, now functioning as gliotransmitters. Whereas the occupancy of the extrasynaptic pre- and postsynaptic receptors by GLU is excitatory (7), the occupancy of these receptors by ATP is inhibitory (8). In addition, neurotransmission is also inactivated by the reuptake of GLU in the membrane of the presynapse mediated by transporter molecules (t) (9). ATP inhibits the presynaptic terminal via occupancy of cognate receptors (prR) temporarily turning off synaptic neurotransmission in the sense of a negative feedback (10). Synaptic information processing is transmitted to neuronal networks activating the synapse again (11). 
parallel, the occupancy of the astrocytic receptors by glutamate also activates $\mathrm{Ca}^{2+}$ within the astrocyte (4). This mechanism exerts the production of glutamate (5) and adenosine triphosphate (ATP) (6) within the astrocyte, now functioning as gliotransmitters. Whereas the occupancy of extrasynaptic pre- and postsynaptic receptors by glutamate is excitatory (7), the occupancy of these receptors by ATP is inhibitory (8). In addition, neurotransmission is also inactivated by the reuptake of glutamate in the membrane of the presynapse mediated by transporter molecules (t) (9). Most importantly, ATP inhibits the presynaptic terminal via occupancy of cognate receptors, temporarily turning off synaptic neurotransmission in the sense of negative feedback (10). Finally, synaptic information processing is transmitted to neuronal networks that can activate the synapse again (11).

In spite of evidence that astrocytes release glutamate by a $\mathrm{Ca}^{2+}$-dependent vesicule mechanism that resembles release from neurons, important differences between glial and neuronal release exist. Glutamate release from astrocytes occurs at a much slower rate than does release from neurons, and it is probably triggered by smaller increases of cytoplasmic $\mathrm{Ca}^{2+}$. Importantly, we apparently deal with different time scales of presynaptic and astrocytic glutamate release. Here, the astrocytic modulatory function of synaptic neurotransmission may occur within seconds or minutes [20]. I hypothesize that the duration from presynaptic activation to the inhibition of synaptic neurotransmission may also be dependent on the amount of astrocytic receptors that must be occupied by glutamate. This mechanism may be based on the occupancy probability of astrocytic receptors by glutamate releases from the presynaptic terminal. In addition, the release of ATP from astrocytes may also be dependent on a comparable mechanism. Accordingly, in GNU's glia may have a temporal boundary-setting function in temporarily turning off synaptic transmission [11,21].

\subsection{Outline of an Astrocyte Domain Organization}

In all mammals, protoplasmic astrocytes are organized into spatially non-overlapping domains that encompass both neurons and vasculature. An astrocyte domain defines a contiguous cohort of synapses that interacts exclusively with a single astrocyte. Synapses within a particular territory are thereby linked via a shared astrocyte partner, independent of a neuronal networking [22]. Single protoplasmic astrocytes operate as a "Lokal Hub" [23]. Figure 4 shows an outline of an astrocyte domain organization. An astrocyte $\left(\mathrm{Ac}_{\mathrm{x}}\right)$ contacts the synapses (Sy) of four neurons $\left(\mathrm{N}_{1} \ldots \mathrm{N}_{4}\right)$ via its processes $\left(\mathrm{P}_{1} \ldots \mathrm{P}_{4}\right)$. Each process is equipped with one to four receptor qualities (Rq). For example, $\mathrm{P}_{1}$ contacts the synapses of $\mathrm{N}_{2}$ exclusively via its receptors of quality a. $\mathrm{P}_{2}$ has already two receptor qualities available (a, b), $\mathrm{P}_{3}$ three receptor qualities (a, b, c) and $\mathrm{P}_{4}$ is able to contact the synapses of $\mathrm{N}_{1}$ via four receptor qualities (a, b, c, d). Astrocyte $\left(\mathrm{Ac}_{\mathrm{x}}\right)$ is interconnected with another astrocyte $\left(\mathrm{Ac}_{\mathrm{y}}\right)$ via gap junctions (g.j.) forming an astrocytic network (syncytium). The neurons per se are also interconnected (neuronal network).

It is experimentally verified that astrocytes can express almost all receptors for important transmitter systems. In certain cases, individual astroglial cells express as many as five different receptor systems linked to $\mathrm{Ca}^{2+}$ mobilization [24]. Each astrocyte territory represents an island made up of many thousands of synapses (about 140.000 in the hippocampal region of the brain, for instance), whose activity is controlled by that astrocyte [25]. On the average, human astrocytes extend 40 large processes radially and symmetrically in all directions from the soma so that each astrocyte supports and modulates the function of roughly two million synapses in the cerebral cortex [22]. Astrocytic receptors are mainly located on the endfeet of the processes. Here, we apparently deal with a high combinational complexity of astrocyte-synaptic interactions [26].

\subsection{Oligodendrocyte-Axonic Interactions}

For normal brain function it is essential that signals pass rapidly between neurons. Oligodendrocytes play an important role in assuring fast neuronal signaling in the central nervous system. By covering neuronal axons with myelin which decreases the effective axonal membrane capacity, they reduce the charge needed to depolarize the axon and hence allow the action potential to travel much faster by saltatory conduction from one node of Ranvier to the next.

During development, oligodendrocytes are generated from precursor cells. These initially differentiate into immature cells that put out processes seeking axons to myelinate, and eventually they form mature cells with parallel processes myelinating up to 30 different axons. The production of myelinated axons requires a precise matching of the number of oligodendrocytes generated to the length of axons to be myelinated [27]. This may be regulated in part by neurotransmitter receptors activated by substances released by active axons. Such interactions may also be important for maintaing the myelination of mature axons. Thus, neurotransmitter receptors play an important role in the life and death of oligodendrocytes $[28,29]$.

Impulse activity in axons affects the development of oligodendrocytes, and thus myelination. Some of the effects of impulse activity inhibit myelination and some stimulate it. Here, the question arises as to how oligodendrocytes know which axons are electrically active. Three mechanisms have been identified that regulate 


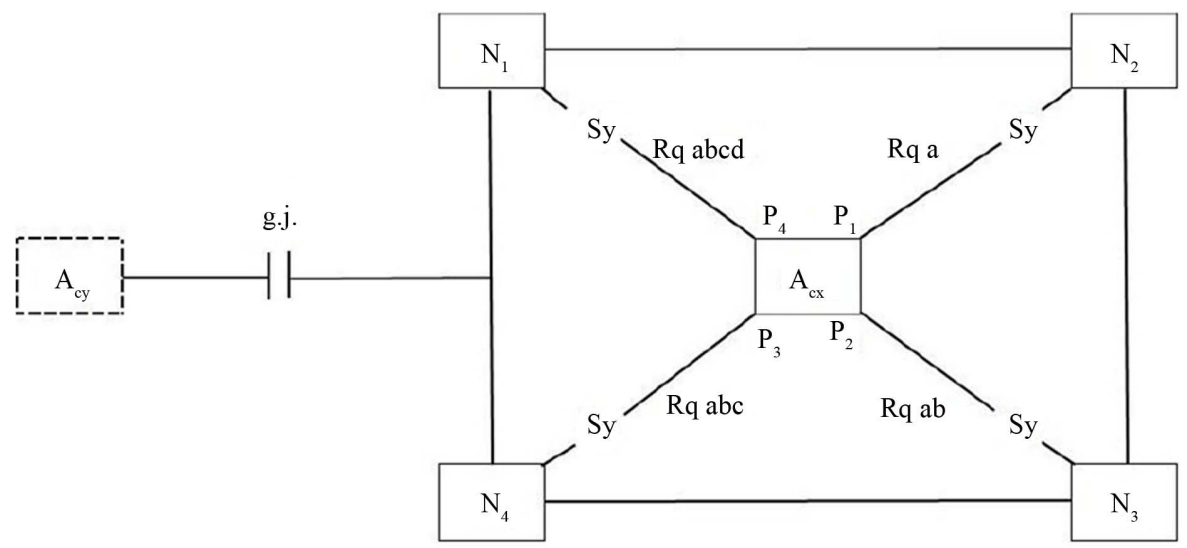

Figure 4. Outline of an astrocyte domain organization. An astrocyte $\left(A_{x}\right)$ is interconnected via four processes $\left(P_{1} \ldots P_{4}\right)$ with the synapses (Sy) of four neurons $\left(\mathrm{N}_{1} \ldots \mathrm{N}_{4}\right)$. Each process is on its endfoot equipped with receptors for the occupancy with neurotransmitters according to a combinational rule [26]. As an example, the receptor $P_{1}$ contacting $N_{2}$ embodies only one receptor quality ( $R q$ a). $P_{2}$ contacts $N_{3}$ with two different receptor qualities $\left(R q\right.$ ab). $P_{3}$ contacts $N_{4}$ with $R q$ abc and $P_{4}$ contacts $\mathrm{N}_{1}$ with $\mathrm{Rq}$ abcd. This simple diagram represents an astrocyte domain. Astrocyte $\left(\mathrm{Ac}_{\mathrm{x}}\right)$ is interconnected with $A c_{\mathrm{y}}$ via gap junctions (g.j.) as shown in more detail in Figure 2 [26].

myelination or the development of myelin forming glia in response to electrical stimulation of axons in vitro [30]. Specific frequencies of electrical impulses control the amount of L1CAM present on unmyelinated axons, a cell adhesion molecule that is necessary for myelination [31]. The neurotransmitter adenosine 5'-triphosphate (ATP) is released from axons and activates receptors on astrocytes, causing them to release the leukemia inhibitory factor that stimulates myelination by mature oligodendrocytes [32]. Adenosine derived from hydrolysis of released ATP promotes oligodendrocyte precursor cell development and thus increases myelination [33]. Moreover, a nonsynaptic mechanism for ATP release from axons has also been identified [34].

\subsection{Glial Modulating Function in Extrasynaptic Neurotransmission}

Rapid signal exchange between astroglia and neurons across the interstitial space emerged as an essential element of synaptic circuit functioning in the brain $[15,35]$. The excitatory neurotransmitter glutamate and the inhibitory neurotransmitter GABA are thought to be the basic transmitters that are regularly from the presynapse occupying cognate receptors on the surface of astrocytes [21]. Recently, extracellular calcium ions $\left(\mathrm{Ca}^{2+}\right)$ have been identified that can report neuronal activity to astroglia [36]. Astrocytes in the hippocampus can respond to activity-induced partial $\mathrm{Ca}^{2+}$ depletion in the extracellular space by generating prominent intracellular waves. The underlying $\mathrm{Ca}^{2+}$ sensing mechanism may involve the opening of the hemichannel connexin 43 in the astrocytic syncytium which in turn triggers the release of adenosine triphosphate (ATP), enhancing the activity of inhibitory interneurons, thus, potentially exerting negative feedback.
This mechanism significantly reduces excessive excitatory activity of neuronal circuits (Figure 5).

Here we deal with a new mechanism of the glial temporal boundary setting function [11]. Whereas astrocytes are capable of turning off synaptic neurotransmission by occupying receptors with gliotransmitters, in extra synaptic neurotransmission an indirect pathway activating inhibitory interneurons through astrocytic ATP release is at work exerting a negative feedback on the presynapse [37]. This mechanism may play an important role in disorders with synaptic hyperexcitability as epilepsy or even non-schizophrenic delusions (see below).

\section{Pathophysiological Model of Schizophrenia}

The core symptoms of schizophrenia can be divided into positive and negative symptoms, with the former including hallucinations, delusions, and disorganization, and the latter including anergia, flattening of affect, and poverty of thought content accompanied by significant disturbances in cognitive function [2]. Hypotheses concerning the etiology of schizophrenia comprise biological, psychological and sociological approaches [38-41]. Generally one can explain delusions and hallucinations in terms of a "loss of ego- or self-boundaries in the sense of an inner/outer confusion" [42-44].

\subsection{Non-Functional Astrocytic Receptors Cause Astrocytic Domain Decomposition}

Let me attempt to show how it may be possible to deduce the main schizophrenic symptoms from an unbalanced tripartite synapse. If the glial receptors are totally nonfunctional and therefore cannot be occupied by neurotransmitters, the system is unbalanced. 


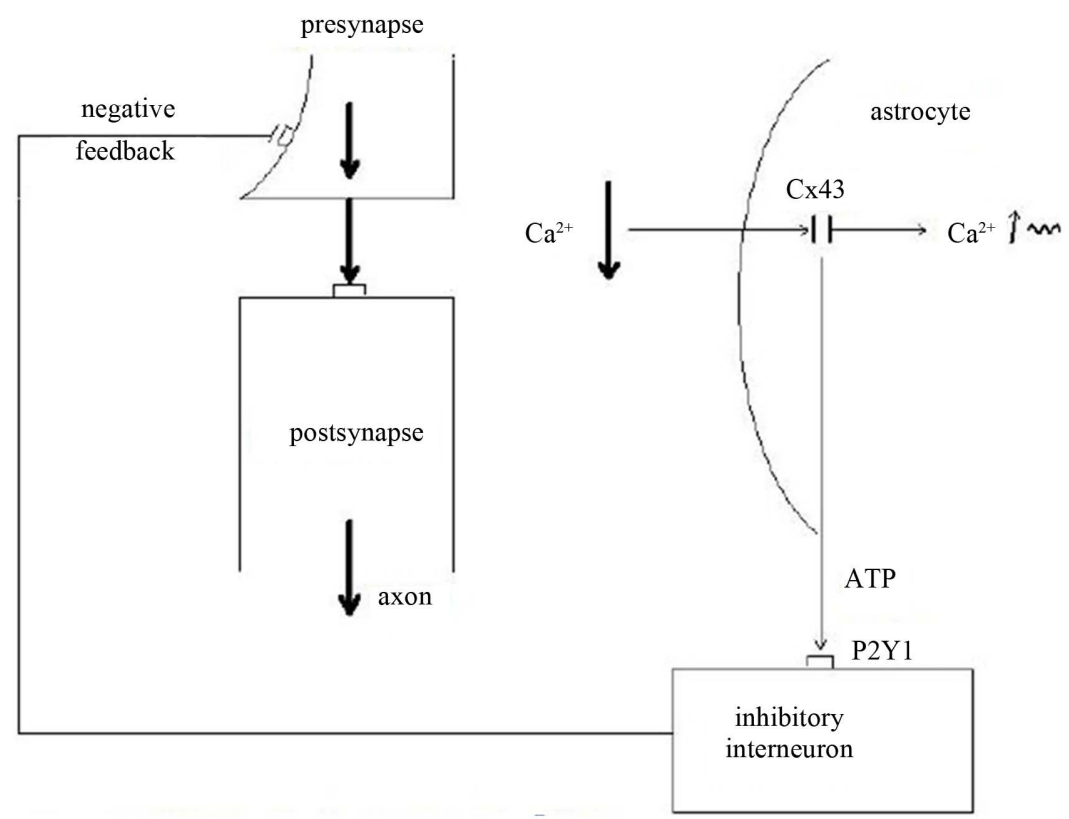

Figure 5. Schematic diagram of extra synaptic glial-neuronal interaction (modified after Torres et al., 2012). The neurotransmission from the presynapse to the postsynapse is hyperexcited (fat arrows) leading to partial depletion of calcium in the extrasynaptic space $\left(\mathrm{Ca}^{2+} \downarrow\right)$. The astrocyte senses the imbalance via connexin $43(\mathrm{Cx} 43)$ hemichannels leading to an increase of intracellular calcium waves $\left(\mathrm{Ca}^{2+} \uparrow \sim\right)$. In parallel, the astrocyte produces adenosine-triphosphate (ATP) that activates P2Y1 receptors on inhibitory interneurons that negatively feed back to the presynapse.

As in Figure 6 depicted, the glial receptors (glR) are non-functional (crosses) and cannot be occupied by neurotransmitters (NT), so that the activation of the gliotransmitters (GT) is impossible. Hence, they cannot negatively feedback to the receptors on the presynapse (prR) and are unable to depolarize the postsynaptic neuron. As a consequence, the glia lose their inhibitory or boundary-setting function and the neural transmitter flux is unconstrained, as the flux of thought on the phenomenological level.

As already described, synaptic glial-neuronal interactions are organized into astrocyte domains. Non-functional astrocytic receptors decompose the glial network from the neuronal one causing a gap between the two. Thus, the term schizophrenia is correct in view of this pathophysiological model. Figure 7 represents a simple diagram of this network decomposition.

Because the astrocyte domain organization may be significant for qualitative information structuring in the brain [26], its decomposition leads to a generalization of information processing in the neuronal networks. Thus, schizophrenics cannot recognize qualitatively different features of subjects and objects but instead they must think in general cases. This cognitive incapability may cause a misinterpretation of a given reality in the sense of delusions and hallucinations. One may argue that a glial determination of neuronal networks into functional units is not necessary because the neuronal system is compartmentalized per se. However, according to my view, there is a qualitative difference between the purely neuronal compartments and the glia-determined domains. Neuronal compartments may be merely functional for information processing, whereas glial-neuronal compartments may in addition have an information-structuring potency that we need for recognizing the qualitative differences between objects and individuals in our environment. That capacity may be lost in schizophrenic patients. Therefore, one can also speak of a loss of conceptual boundaries in schizophrenia.

Let me now deduce the main symptoms of schizophrenia from unbalanced tripartite synapses caused by nonfunctional glial receptors. Table 1 shows the basic schizophrenic symptoms [45] that may be caused by a loss of conceptual boundaries. This disorder can affect cognitive processes such as thinking. If a schizophrenic patient is unable to delimit conceptual boundaries among words, thoughts, or ideas with different meanings, then meaningless word constructs (neologisms) or disorganized speech are the typical phenomenological manifestations, called "thought disorder".

From an ontological point of view, delusions are the consequence of the loss of boundaries between the self and the others (nonselves). Here, the self is defined as a living system capable of self-observation [46]. One could also say that our brain embodies a distinct ontological locus of self-observation. Everything taking place in the brains of schizophrenic patients is reality because they cannot differentiate between their inner world and the 


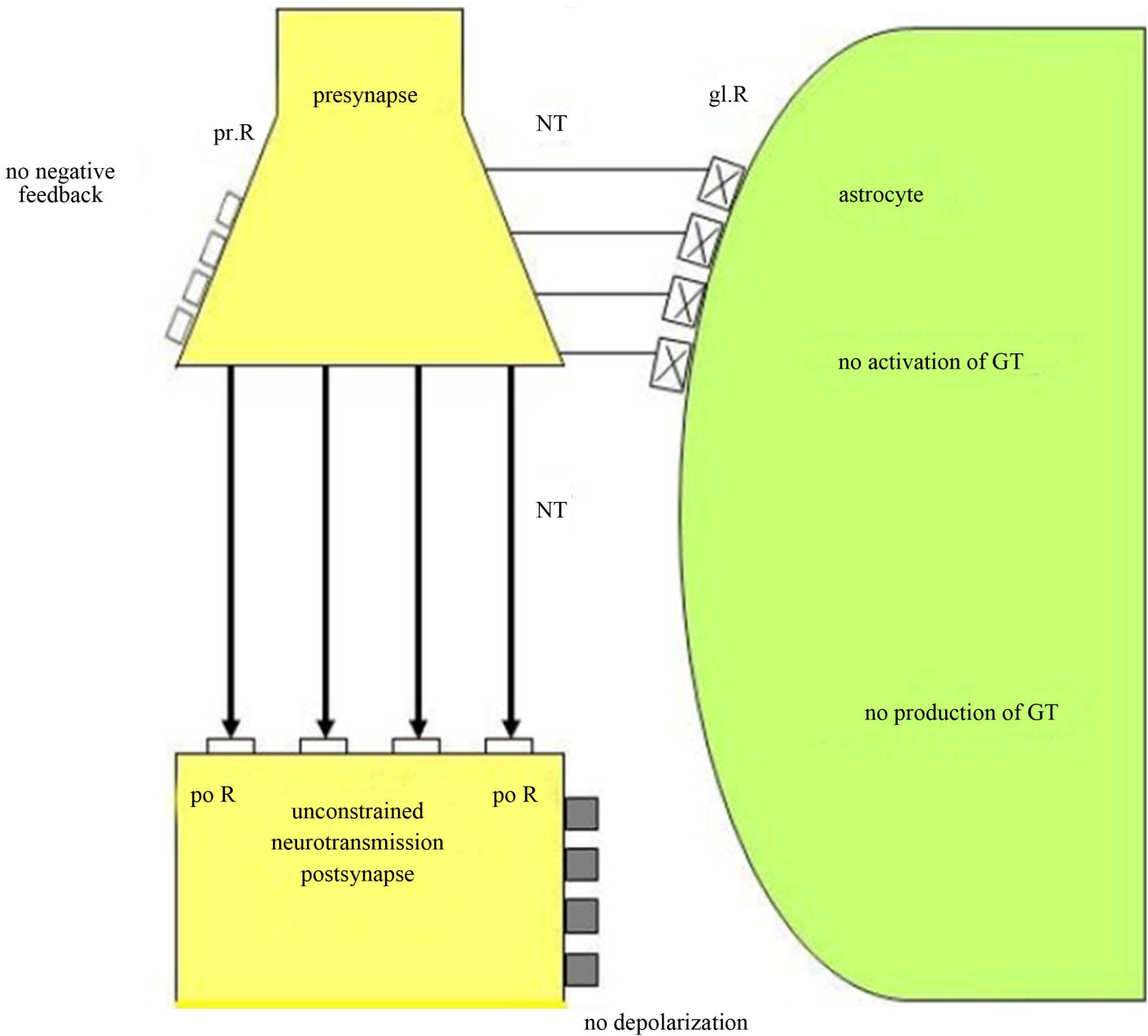

Figure 6. Unconstrained neurotransmission in tripartite synapses may cause schizophrenia. Non-functional glial receptors (glR), depicted by crosses, cannot be occupied by neurotransmitters (NT). Since the activation and production of gliotransmitters (GT) is not possible, glia do not negatively feed back to the presynaptic receptors (prR) and cannot depolarize the postsynaptic neuron. This severe synaptic disturbance leads to an unconstrained neurotransmission (fat arrows).

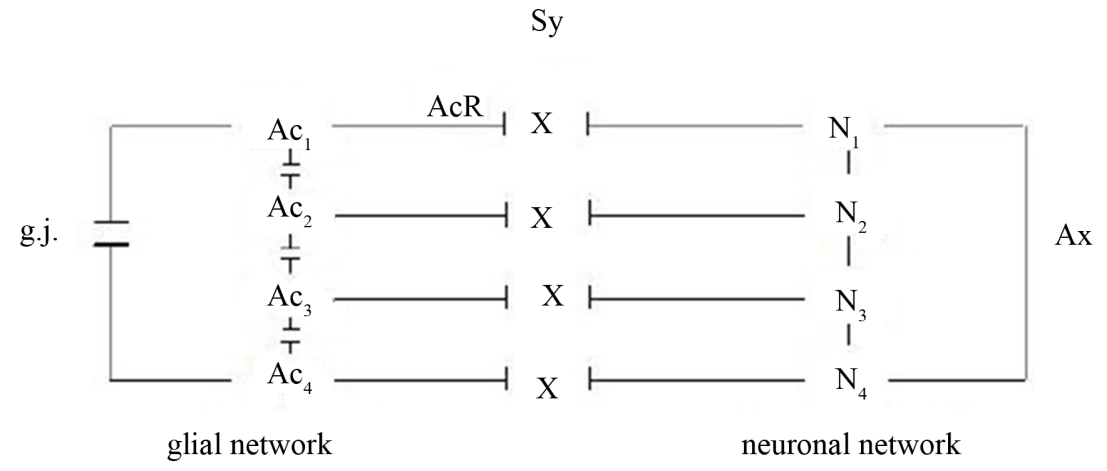

Figure 7. Gaps between the glial and the neuronal networks caused by non-functional astrocytic receptors (crosses). Four astrocytes $\left(\mathrm{Ac}_{1} \ldots \mathrm{Ac}_{4}\right)$ are interconnected via gap junctions (g.j.) building a glial network. Four neurons $\left(\mathrm{N}_{1} \ldots \mathrm{N}_{4}\right)$ are interconnected via axons (Ax) building a neuronal network (dendrites not shown). The synaptic interactions between the glial and neuronal networks are interrupted, since the astrocytic receptors (AcR) are nonfunctional (crosses). 
Table 1. Interpretation of basic schizophrenic symptoms.

\begin{tabular}{cc}
\hline Loss of boundaries & Symptoms \\
Conceptual & Thought disorder \\
Ontological & Delusions \\
Perceptive & Hallucinations \\
Motoric & Catatonic symptoms \\
Emotional & Affective flattening \\
\hline
\end{tabular}

If the loss of boundaries concerns concepts, a thought disorder results. In the case of a loss of ontological boundaries among the self and the others (Non-Selves), delusions occur. This loss of ontological boundaries can also affect the perception system in the sense of hallucinations. The loss of boundaries among motor modules shows up in catatonic phenomena. If the boundaries between emotional qualities are lost, affective flattening is the typical symptom [44].

outer world. Therefore, they cannot see ontological differences between the selves and the nonselves. This loss of ontological boundaries may lead to a delusional misinterpretation of reality.

Hallucinations may be caused by the same disorder. However, the perception systems are phenomenologically affected. A schizophrenic who hears the voice of a person in his head is absolutely convinced that this person is really speaking to him. The loss of ontological boundaries or inner/outer confusion shows its phenomenological manifestation in the auditory system. Such a disorder can also occur in other sensory systems.

If the loss of boundaries affects the motor system in the brain, the symptomatology is called catatonia. A state of catatonic agitation in which a disinhibited discharge of nearly all motor systems occurs is an expression of motor generalization with raging and screaming as behavioral components. One could also say that the brain's inability to constrain information processing among motor modules appears in catatonic phenomena. Hence, the catatonic type of schizophrenia represents a serious disorder of motor behavior. Typical symptoms are excessive motor activity and motoric immobility (stupor). Both phenomena appear to be purposeless and not influenced by external stimuli. In such a catatonic state, a patient is unable to communicate. He or she cannot see the other. Everything that happens takes place in the brain of the patient.

Affective flattening is regarded as a negative schizophrenic symptom [47]. This symptom can also be explained as a loss of boundary setting. The different affective or emotional qualities cannot be produced within the brain, and the communication of feelings is disturbed as a result [48].

\subsection{Some Genetic Considerations}

There is growing evidence of disease-related altered astrocyte gene expression. These findings suggest an im- balance of glutamate-glutamine cycle in the communication of neurons and astrocytes [49]. There is also evidence for a broad involvement of astrocytes in other aspects of the pathophysiology in schizophrenia [7]. However, if we focus on nonfunctional receptor proteins on astrocytes, an aberrant splicing may represent a genetic candidate mechanism. Aberrant or non-splicing causes truncated or chimeric proteins such that receptor occupancy is not possible. What the mechanisms of aberrant splicing concerns, significant findings have been reported [50]. Nonsense, missense, and even synonymous mutations can induce aberrant skipping of the mutant exon, producing nonfunctional proteins. If the exchange of nucleotides generates a synonymous codon that represents the same aminoacid as the original triplet, one speaks of a silent mutation. These mutations have erroneously been classified as nonpathogenic, but are now recognized as affecting the splicing machinery resulting in defective proteins. Aberrant splicing may play a decisive role in the pathophysiology of various diseases [51]. Why not in schizophrenia, as I hypothesized nearly a decade ago?

Recently, gene losses in the human genome have been identified [52]. These loss-of-function variants are located in human protein-coding genes. Somatic mutation and genomic variation may also play a role in neurological disease [53]. Since the first comprehensive, genomewide catalogue of variants likely to disrupt protein-coding genes is now available, this genetic approach could also be promising for the identification of nonfunctional receptors on astrocytes in brains with schizophrenia. Moreover, epigenetic factors may also play an important role. Epigenetics broadly refers to heritable changes in phenotype or gene expression caused by mechanisms other than changes in the underlying primary DNA sequence. Several major types of epigenetic mechanisms are DNA methylation, genomic imprinting, histone modifications, and expression control by noncoding RNA. Recent data suggest the influence of these epigenetic alterations in schizophrenia [54].

\subsection{Unconstrained Neurotransmission May Cause Demyelination}

Generally, in pathological conditions neurotransmitters can be released excessively, damaging the cells they normally act on. Since oligodendrocytes have receptors for the various transmitters, neurotransmitter excess can cause demyelination [28]. Already in 1977 a study was published documenting severe breakdown of myelin in dogs injected with myelin [55]. In the grey matter of the brain the death of neurons in pathological conditions is often caused by a rise of extracellular glutamate concentration activating NMDA receptors and causing an excessive rise of $\mathrm{Ca}^{2+}$. Glutamate can also damage white matter oligodendrocytes. Concentrations of glutamate 
which alone are not toxic sensitize oligodendrocytes to subsequent complementary attack that inserts membrane attack complexes into the oligodendrocyte, allowing a toxic $\mathrm{Ca}^{2+}$ influx to occur [56].

In line with the presented synaptic model of the pathophysiology of schizophrenia, an unconstrained flux of neurotransmitters occurs. This may hold for all the various neurotransmitter types. This unconstrained flux of neurotransmitters may affect oligodendrocytes either by flooding of their cognate receptors on oligodendrocytes, exerting a toxic $\mathrm{Ca}^{2+}$ influx, or via a hyperactivation of axons with an excess of axonic ATP production and a consequent toxic effect on oligodendrocytes [29] (Figure 8). In addition, ATP released from axons cannot activate astrocytic receptors, since they do not function. Therefore, a stimulation of myelination by mature oligodendrocytes is not possible. These pathological mechanisms may cause demyelination, as observed in brains with schizophrenia [57,58]. Note, although decreased expression of oligodendrocyte-related genes has been identified [59], it seems implausible that genetics alone could account for demyelination in schizophrenia [60].

Moreover, the permanent hyperactivation of axons may also impair them so that neuronal networks disconnect. This may represent a possible mechanism that could at least in part be responsible for the disconnection of neuronal networks identified in brains with schizophrenia
[29,59].

\subsection{Loss of Oligodendrocytes May Cause a Decay of Gap Junctions in the Panglial Syncytium Responsible for Memory Impairment}

Since gap junctions between astrocytes and oligodendrocytes are heterotypic, composed of special astrocytic connexins and different oligodendrocytic connexins, a loss of oligodendrocytes disrupts astrocyte-oligodendrocytic gap junctions in the panglial syncytium, leading to a "leaky" syncytium (Figure 9).

Gap junctions form plaques that may embody memory structures [61]. Therefore, a loss of gap junctions may impair memory. In addition, genetic or (and) exogenous disorders can affect gap junctional plaque formation which may be the case in schizophrenia. In this context one could speak of a syncytiopathy in schizophrenia [62]. However, memory impairment may also be caused by a disorganization of neuronal networks [63].

\subsection{Schizophrenic Dysintentionality Based on a Severe Disorder of Glial-Neuronal Interactions}

According to Frith [64], schizophrenia can be explained

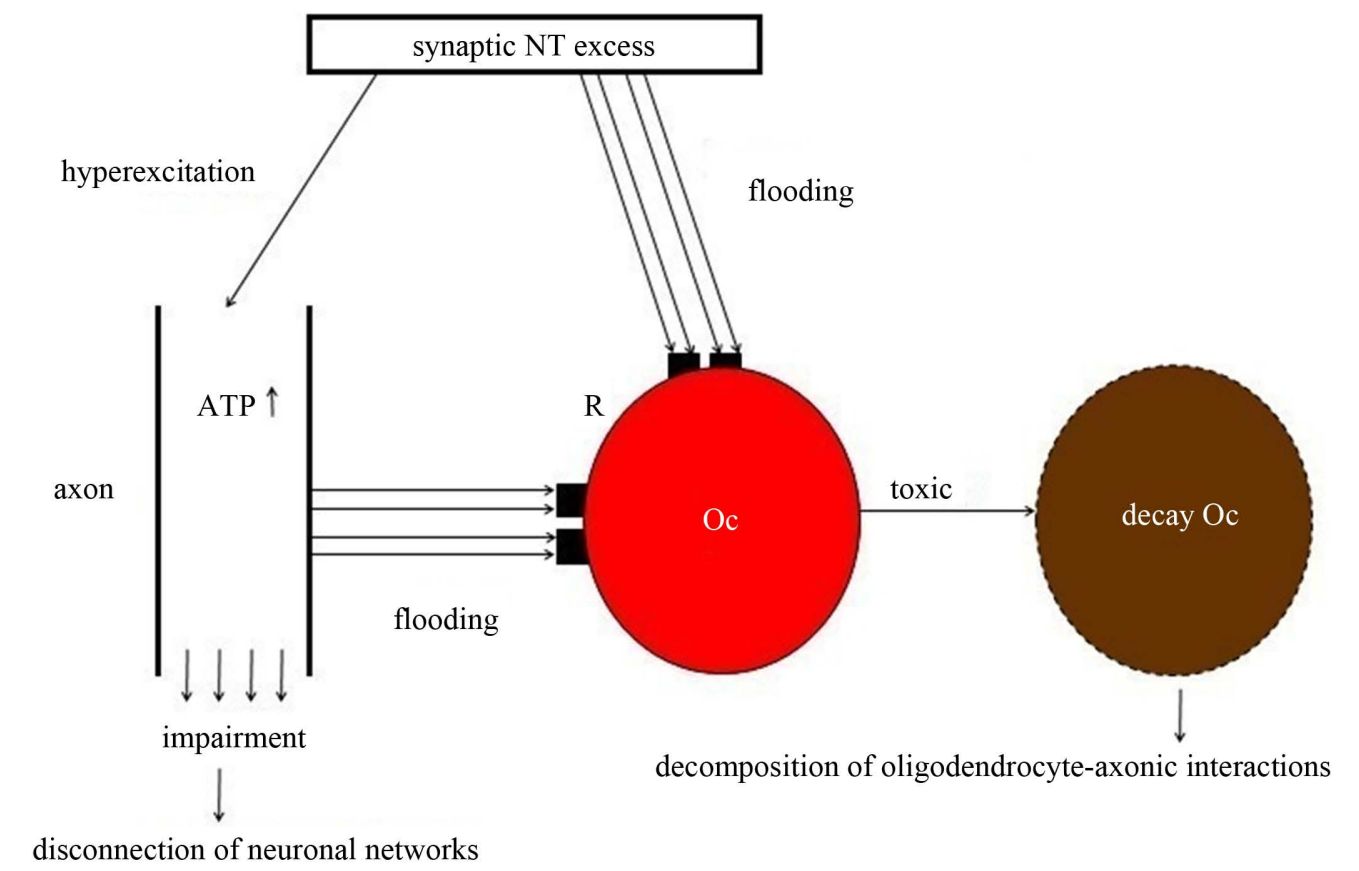

Figure 8. Schematic diagram of the excess of neurotransmitters that may cause the loss of oligodendrocytes and the decomposition or disconnection of both the oligodendrocyte-axonic interactions and neuronal networks. A synaptic neurotransmitter (NT) excess hyperexcites the axon and floods the cognate receptors (R) on the oligodendrocyte (Oc). In parallel, a non-synaptic ATP excess occurs, also flooding R. This flooding of NT and ATP exerts a toxic effect on the Oc, leading to its decay. These mechanisms may be responsible for the decomposition of oligodendrocyte-axonic interactions and the disconnection of neuronal networks [29]. 
panglial syncytium

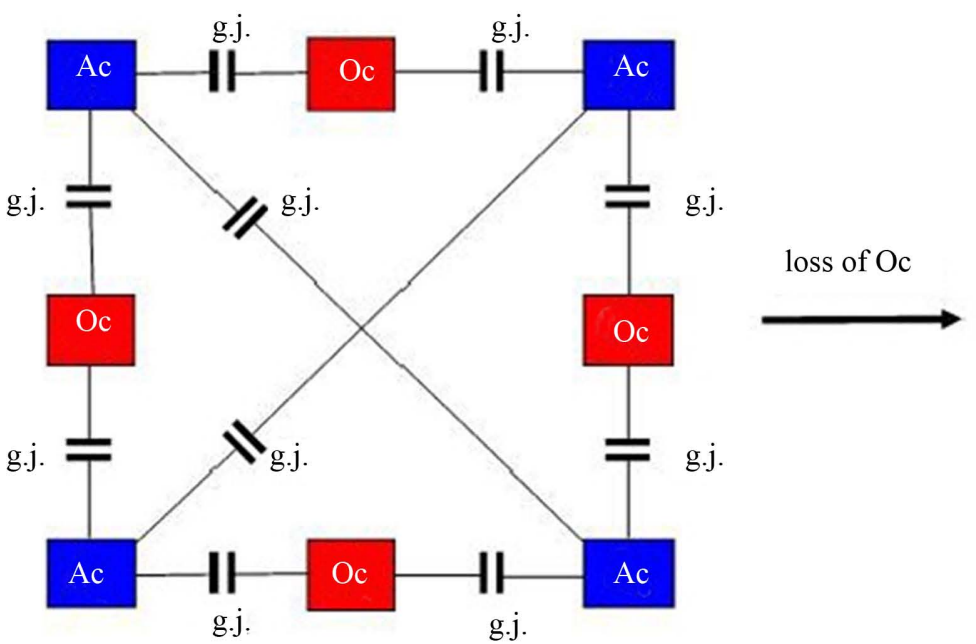

"leaky" panglial syncytium

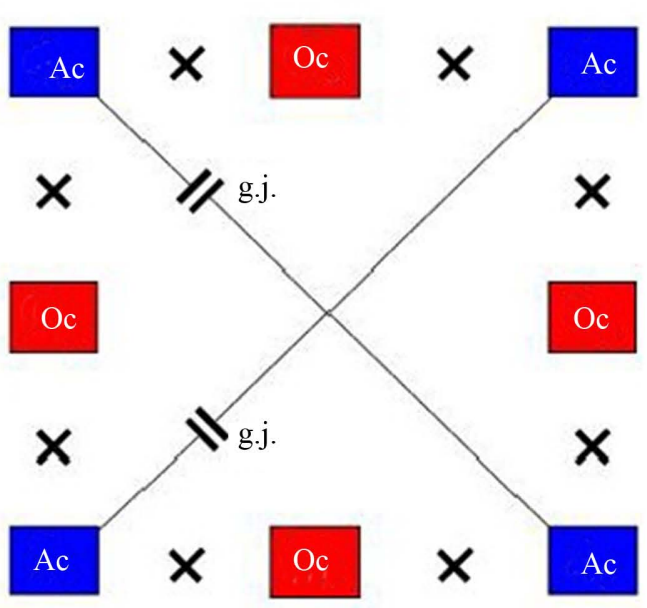

Figure 9. Loss of oligodendrocytes disconnects the panglial syncytium responsible for memory impairment in schizophrenia. On the left, a panglial syncytium composed of astrocytes (Ac) and oligodendrocytes (Oc) interconnected via gap junctions (g.j.). A loss of Oc may cause a decay of Oc-Ac gap junctions (crosses). Such a "leaky” panglial syncytium may be responsible for memory impairment.

by a failure to integrate the intention to act with the perceptual registration of the consequences of that action. At a neurobiological level this integrative abnormality might correspond to a failure to integrate signals from the (intentional) prefrontal regions and the (perceptual) temporal cortices. However, this is a pure neuronal view excluding the glial cell system. In further elaborating my theory of glial-neuronal interaction, I have hypothesized that the intentional or action programs of the brain may be generated in the panglial syncytium [12]. Based on a formal model it can be shown how glial syncytia compute in a highly combinatorial manner cycles of various lengths via gap junctions. These cycles are transferred in tripartite synapses to the neuronal system [65]. The neuronal system tests these intentional programs with regard to their feasibility in the environment. In feeding back the feasibility of intentional programs to the glial syncytium, learning processes can occur.

For the clinically experienced psychiatrist it is evident that patients with schizophrenia are unable both to test their delusional programs and to realize these unrealistic intentions in the environment. I have named this disorder "schizophrenic dysintentionality" [66]. But let us consider the elementary pathophysiological mechanisms of schizophrenia as proposed. First of all, there is a break of information processing between the glial system and the neuronal system in tripartite synapses and also in the "orthogonal" oligodendrocyte-axonic interaction. In this view, the term schizophrenia (split of consciousness) is appropriate. In other words: a patient with schizophrenia is permanently stressed by a world of intentions that cannot be mediated via tripartite synapses to the neuronal system for reality testing. Such considerations could be explanatory with concern to recent findings of abnormalities in the white matter of the brain [67]. Supposing that a patient is under permanent pressure to realize his/ her intentional programs generated in the panglial syncytium, then the normal apoptosis could be accelerated or mutations in astrocytes and in the oligodendrocyte-myelin system could be activated. The effect is a decrease in white matter. Considering the loss of oligodendrocytes which are normally interconnected with astrocytes via gap junctions, the decay of oligodendrocytes must also destruct the panglial syncytium in the sense of an increasing loss of gap junctions. This loss of gap junctions may again destruct the capacity of the panglial syncytium to generate intentional programs.

Many patients with schizophrenia become increasingly psychobiologically exhausted in the chronic course of their illness, which is called schizophrenic residuum. Of course, the frequently observed disorders in neuronal networks may also play a role, but the destruction of the panglial syncytium leading to a dysintentionality per se, may be basically responsible for the negative view of life, as typically seen in the schizophrenic residuum. Most impressively, if the prefrontal cortex is severely affected, these patients are incapable of planning. Therefore, what they want is merely the satisfaction of simple biological needs (eating, drinking, smoking, getting money to buy something, etc.). One could also say that with the destruction of the panglial syncytium all kinds of destiny are broken down as well. 
How could astrocytes react to this disaster? Reactive astrocytosis may be a compensatory attempt. Reactive astrocytosis occurs prominently in response to all forms of CNS injury or disease. Recent studies point to the role of reactive astrocytes in helping to limit tissue degeneration and preserve function after CNS injury [68]. So why not in schizophrenia? If one interprets the degeneration of the panglial syncytium caused by stress as a functional brain injury, then reactive astrocytosis may here exert the same mechanism. However, in schizophrenia astrocytosis may not only react to injuries of the neuronal system, but also attempt to generate a new astrocytic syncytium in reaction to the degeneration of the panglial syncytium. In this way the patient can generate intentional programs and keep a "touch of destiny" alive. This conjecture is experimentally testable, if one compares the degree of dysintentionality-defined as the ability to produce intentions or plans — of schizophrenic patients with and without reactive astrocytosis.

Finally, reactive astrocytosis can be seen in the light of the Astrocentric Hypothesis [61]. According to this hypothesis, astrocytes represent the core cells in the brain that not only control the glial-neuronal interaction, but also determine the functions within the panglial syncytium. Therefore, astrocytes may be capable-at least- to attempt repairing dysfunctions in the panglial syncytium, as may be the case in schizophrenia. Admittedly, the pertinent findings in brains with schizophrenia are contradictory. However, astrocytosis (astrogliosis) may not occur widespread in the brain [7], but it could represent a local phenomenon.

\subsection{Pathophysiology of Non-Schizophrenic Delusions}

The pathophysiological mechanism of non-schizophrenic delusions may significantly differ from schizophrenic delusions. Let us focus on the extrasynaptic fluid, especially $\mathrm{Ca}^{2+}$ ions, in information transmission. Astrocytes sense a decrease of $\mathrm{Ca}^{2+}$ in the extrasynaptic space via Cx43 hemichannels and produce ATP that activates inhibitory interneurons, which negatively feed back to the presynapse. I hypothesize that if $\mathrm{Ca}^{2+}$ is totally exhausted (e.g. by stress), the negative feedback mechanism cannot be generated. The exhaustion of $\mathrm{Ca}^{2+}$ may be caused by hyperexcitable neurons flooding the synaptic neurotransmission. Importantly, the astrocytic receptors are normally expressed, but they cannot cope with the synaptic neurotransmitter flooding without an extrasynaptically generated negative feedback. Thus, in nonschizophrenic delusions an unconstrained information flux may also occur, but astrocytes per se may not be affected. Here, the brain operates in a holistic and not schizophrenic way. This pathophysiological mechanism could explain why patients with paranoid psychoses can mostly be successfully treated with antipsychotic drugs. Their blockade of postsynaptic receptors constrains neurotransmission such that astrocytes can work normally again. Although the neurons may remain hyperexcited, the $\mathrm{Ca}^{2+}$ depletion is sufficient to activate the negative feedback mechanism described. In parallel, the astrocytic receptors are again able to modulate synaptic information processing, since they can cope with the amount of neurotransmitters and temporarily turn off synaptic information processing based on a negative feedback mechanism.

\section{Conclusions}

My model of impaired glial-neuronal interactions in schizophrenia is based on the core hypothesis that nonfunctional astrocytic receptors may cause an unconstrained synaptic information flux such that glia lose their modulatory function in tripartite synapses (Figure 10).

This may lead to a generalization of information processing in the neuronal networks responsible for delusions and hallucinations on the behavioral level. In this acute paranoid stage of schizophrenia, nonfunctional astrocytic receptors or their loss decompose the astrocyte domain organization with the effect that a gap between the neuronal and the glial networks arises. If the illness deteriorates, the permanent synaptic neurotransmitter flux may additionally impair the oligodendrocyte-axonic interactions, followed by a "creeping" decay of oligodendroglia, axons, and glial gap junctions responsible for severe cognitive impairments. Here we may deal with aftereffects caused by the basic fault of information processing in tripartite synapses. Importantly, the same may hold true what the neuroinflammatory hypothesis [69] concerns. The activation of microglia observed in brains with schizophrenia could represent a reaction to the decay of nervous tissue described and not a primary pathophysiological mechanism of schizophrenia.

Currently, post mortem brains with schizophrenia are investigated with Storm microscopy, a method that achieves resolution below the optical diffraction limit (University of Applied Sciences, Linz, Austria). Whereas in the prefrontal cortex of normal brains big amounts of receptors located on the processes of astrocytes are found, in brains with schizophrenia astrocytic receptors cannot be identified in this region. Since these preliminary results concern only serotonergic receptors in the prefrontal cortex, further investigations of other receptor types on astrocytes in pertinent brain regions is necessary. Should it be possible to verify the central role of astrocytic receptors in the pathophysiology of schizophrenia, then a diagnostic marker would be available, if an in vivo iden- 


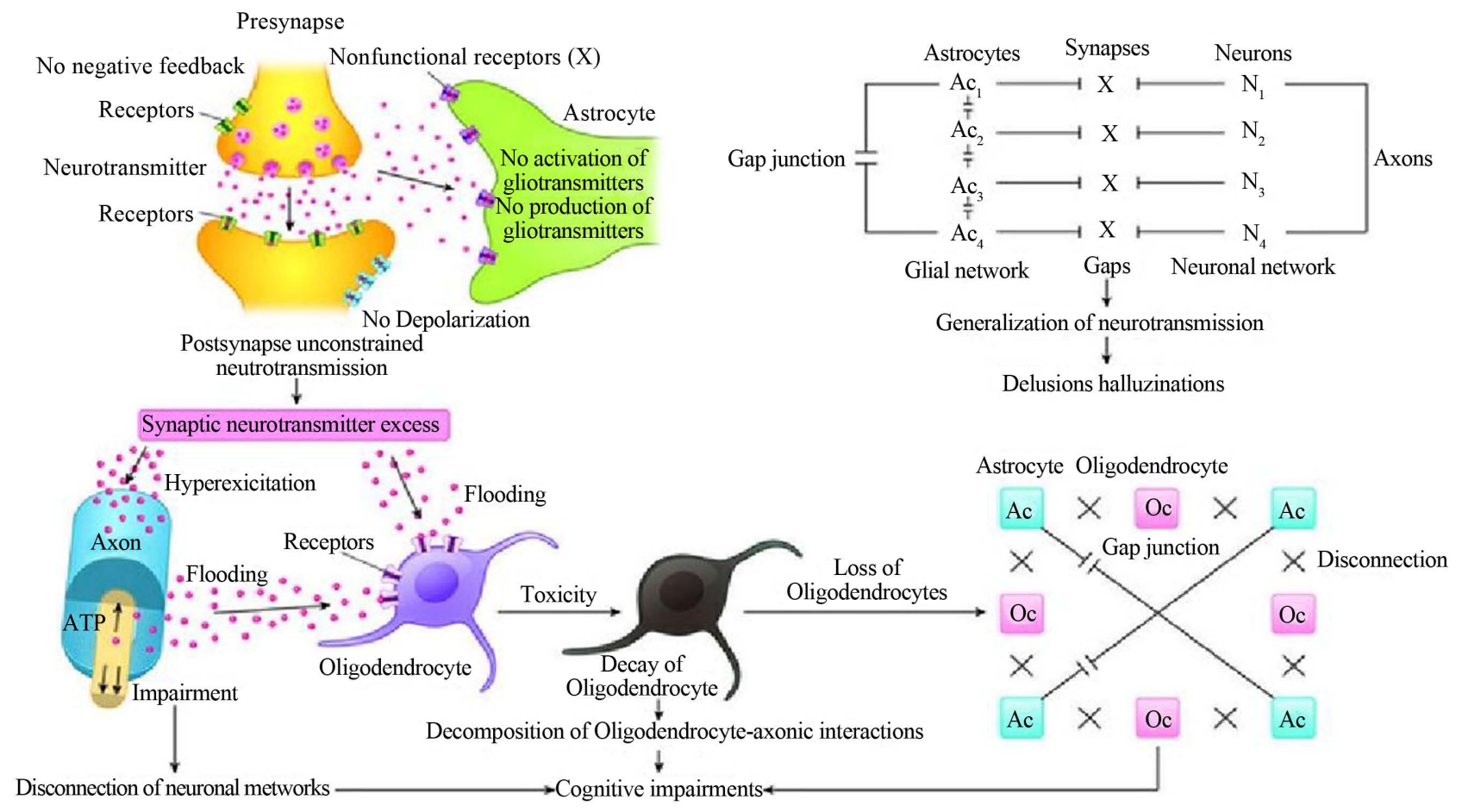

Figure 10. Overview of basic glial impairments in their interactions with the neuronal system, responsible for the pathophysiology of schizophrenia.

tification is possible as well.

\section{Acknowledgements}

This paper is dedicated to my friend and great neuroscientist Gerhard Werner ( $\dagger$ ), University of Austin, Texas. I am also grateful to Birgitta Kofler-Westergren for preparing the final version of this paper.

\section{REFERENCES}

[1] V. Parpura, M. T. Heneka, V. Montana, S. H. Oliet, et al., "Glial Cells in (Patho)physiology," Journal of Neurochemistry, Vol. 121, No. 1, 2012, pp. 4-27. http://dx.doi.org/10.1111/j.1471-4159.2012.07664.x

[2] H. Meltzer, "Multiple Neurotransmitters Involved in Antipsychotic Drug Action,” In: S. Kapur and Y. Lecrubier, Eds., Dopamine in the Pathophysiology and Treatment of Schizophrenia, Martin Dunitz, London, 2003, pp. 177205. http://dx.doi.org/10.3109/9780203427347-10

[3] S. Berge and T. Koenig, "Cerebral Disconnectivity: An Early Event in Schizophrenia,” Neuroscientist, Vol. 14, No. 1, 2008, pp. 19-45.

[4] K. J. Friston, “The Disconnection Hypothesis," Schizophrenia Research, Vol. 30, No. 2, 1998, pp. 115-125. http://dx.doi.org/10.1016/S0920-9964(97)00140-0

[5] P. Erdi, B. Flaugher, T. Jones, B. Ujfalussy, L. Zalanyi and V. A. Diwadkar, "Computational Approach to Schizophrenia. Disconnection Syndrome and Dynamicalpharmacology," AIP Conference Proceedings, Varenna, 24-28 September 2007, pp. 65-87. http://dx.doi.org/10.1063/1.2965102

[6] K. I. Vadakkan, "A Structure-Function Mechanism for Schizophrenia,” Frontiers in Psychiatry, Vol. 3, No. 108, 2012. http://dx.doi.org/10.3389/fpsyt.2012.00108

[7] H. G. Bernstein, J. Steiner and B. Bogerts, "Glial Cells in Schizophrenia: Pathophysiological Significance and Possible Consequences for Therapy," Expert Review Neurotherapy, Vol. 9, No. 7, 2009, pp. 1059-1071. http://dx.doi.org/10.1586/ern.09.59

[8] X. Di, R. C. Chan and Q. Gong, "White Matter Reduction in Patients with Schizophrenia as Revealed by VoxelBased Morphometry: An Activation Likely Hood Estimation Meta-Analysis," Progress in Neuropsychopharmacology, Biology and Psychiatry, Vol. 33, No. 8, 2009, pp. 1390-1394.

[9] M. Kyriakopoulos, R. Perez-Iglesias, J. B. Woolley, R. A. Kamaan, N. S. Vyas, G. J. Barker, S. Frangou and P. K. McGuire, "Effect of Age at Onset of Schizophrenia on White Matter Abnormalities," British Journal of Psychiatry, Vol. 195, No. 4, 2009, pp. 346-353. http://dx.doi.org/10.1192/bjp.bp.108.055376

[10] A.E. Steffek, "The Role of Astrocytes in the Pathophysiology of Schizophrenia,” Dissertation, University of Michigan, Ann Arbor, 2007, 159 p.

[11] B. Mitterauer, “An Interdisciplinary Approach towards a Theory of Consciousness,” Biosystems, Vol. 45, No. 2, 1998, pp. 99-121. http://dx.doi.org/10.1016/S0303-2647(97)00070-1

[12] B. Mitterauer, "Where and How Could Intentional Programs Be Generated in the Brain? A Hypothetical Model Based on Glial-Neuronal Interactions,” Biosystems, Vol. 
88, No. 1-2, 2007, pp. 101-112.

http://dx.doi.org/10.1016/j.biosystems.2006.04.003

[13] H. Kettenmann and B. R. Ransom, “Neuroglia,” Oxford University Press, Oxford, 2005.

[14] M. M. Halassa, T. Fellin and P. G. Haydon, "Tripartite Synapses: Roles for Astrocytic Purins in the Control of Synaptic Physiology and Behavior," Neuropharmacology, Vol. 57, No. 4, 2009, pp. 343-346. http://dx.doi.org/10.1016/j.neuropharm.2009.06.031

[15] D. A. Rusakov, "Depletion of Extracellular $\mathrm{Ca}^{2+}$ Prompts Astroglia to Modulate Synaptic Network Activity," Science Signalling, Vol. 5, No. 208, 2012, p. 4. http://dx.doi.org/10.1126/scisignal.2002799

[16] R. Dermietzel and D. C. Spray, "From Neuro-Glue to Glia: A Prologue,” Glia, Vol. 24, No. 1, 1998, pp. 1-7. http://dx.doi.org/10.1002/(SICI)1098-1136(199809)24:1< 1::AID-GLIA1>3.0.CO;2-A

[17] A. Araque, V. Parpura, R. P. Sanzgiri and P. G. Haydon, "Tripartite Synapses: Glia, the Unacknowledged Partner," Trends in Neuroscience, Vol. 22, No. 5, 1999, pp. 208215. http://dx.doi.org/10.1016/S0166-2236(98)01349-6

[18] B. R. Ransom and Z. Ye, "Gap Junctions and Hemichannels,” In: H. Kettenmann and B. R. Ransom, Eds., Neuroglia, Oxford University Press, Oxford, 2005, pp. 177189.

[19] E. A. Newman, “Glia and Synaptic Transmission,” In: H. Kettenmann and B. R. Ransom, Eds., Neuroglia, Oxford University Press, Oxford, 2005, pp. 355-366.

[20] D. Stellwagen and R. C. Malenka, "Synaptic Scaling Mediated by Glial TNF-Alpha,” Nature, Vol. 440, No. 7087, 2006, pp. 1054-1059. http://dx.doi.org/10.1038/nature04671

[21] D. S. Auld and R. Robitaille, "Glial Cells AND Neurotransmission: An Inclusive View of Synaptic Function,” Neuron, Vol. 40, No. 2, 2003, pp. 389-400. http://dx.doi.org/10.1016/S0896-6273(03)00607-X

[22] N. A. Oberheim, X. Wang, S. Goldman and M. Nedergaard, "Astrocytic Complexity Distinguishes the Human Brain,” Trends in Neuroscience, Vol. 29, No. 10, 2006, pp. 547-553. http://dx.doi.org/10.1016/j.tins.2006.08.004

[23] A. Pereira and F. A. Furlan, "Astrocytes and Human Cognition: Modeling Information Integration and Modulation of Neuronal Activity," Progress in Neurobiology, Vol. 92, No. 3, 2010, pp. 405-420. http://dx.doi.org/10.1016/j.pneurobio.2010.07.001

[24] H. Kettenmann and C. Steinhäuser, "Receptors for Neurotransmitters and Hormones,” In: H. Kettenmann and B. R. Ransom, Eds., Neuroglia, Oxford University Press, Oxford, 2005, pp. 131-145.

[25] M. Santello and A. Volterra, "Astrocytes as Aide-Mémoires,” Nature, Vol. 463, No. 7278, 2010, pp. 169-170. http://dx.doi.org/10.1038/463169a

[26] B. Mitterauer, "Significance of the Astrocyte Domain Organization for Qualitative Information Structuring in the Brain," Advances in Bioscience and Biotechnology, Vol. 1, 2010, pp. 391-397. http://dx.doi.org/10.4236/abb.2010.15052

[27] R. D. Fields, A. Araque, H. Johansen-Berg, S. Lien, et al.,
“Glial Biology in Learning and Cognition,” Neuroscientist, 2013. http://dx.doi.org/10.1177/1073858413504465

[28] R. Káradóttir and D. Attwell, "Neurotransmitter Receptors in the Life and Death of Oligodendrocytes," Neuroscience, Vol. 145, No. 4, 2007, pp. 1426-1438. http://dx.doi.org/10.1016/j.neuroscience.2006.08.070

[29] B. Mitterauer and B. Kofler-Westergren, "Possible Effects of Synaptic Imbalances on Oligodendrocyte-Axonic Interactions in Schizophrenia: A Hypothetical Model," Frontiers in Psychiatry, Vol. 2, 2011, p. 15. http://dx.doi.org/10.3389/fpsyt.2011.00015

[30] R. D. Fields, “Change in the Brain’s White Matter,” Science, Vol. 330, No. 6005, 2010, pp. 768-769. http://dx.doi.org/10.1126/science.1199139

[31] B. Stevens, S. Tanner and R. D. Fields, "Control of Myelination by Specific Patterns of Neural Impulses,” Journal of Neuroscience, Vol. 18, No. 22, 1998, pp. 9303-9311.

[32] T. Ishibashi, K. A. Dakin, B. Stevens, P. R. Lee, S. V. Kozlov, C. L. Stewart and R. D. Fields, "Astrocytes Promote Myelination in Response Toelectrical Impulses," Neuron, Vol. 49, No. 6, 2006, pp. 823-832. http://dx.doi.org/10.1016/j.neuron.2006.02.006

[33] B. Stevens, S. Porta, L. L. Haak, V. Gallo and R. D. Fields, "Adenosine, a Neuron-Glial Transmitter Promoting Myelination in the CNS in Response to Action Potentials,” Neuron, Vol. 36, No. 5, 2002, pp. 855-868. http://dx.doi.org/10.1016/S0896-6273(02)01067-X

[34] R. D. Fields and Y. Ni, "Nonsynaptic Communication through ATP Release from Volume-Activated Anion Channels in Axons,” Science Signalling, Vol. 3, No. 142, 2010, p. ra73.

[35] F. F. De-Miguel and K. Fuxe, "Extrasynaptic Neurotransmission as a Way of Modulating Neuronal Functions," Frontiers in Physiology, Vol. 3, 2012, p. 16.

[36] A. Torres, F. Wang, Q. Xu, T. Fujita, R. Dobrowolski, K. Willecke, T. Takano and M. Nedergaard, "Extracellular $\mathrm{Ca}^{2+}$ Acts as a Mediator of Communication from Neurons to Glia,” Science Signaling, Vol. 5, No. 208, 2012, p. ra8.

[37] G. Werner and B. J. Mitterauer, "Neuromodulatory Systems,” Frontiers in Neural Circuits, Vol. 7, 2013, p. 36.

[38] W. T. Carpenter and R. W. Buchanan, "Schizophrenia: Introduction and Overview," In: H. J. Kaplan and B. J. Sadock, Eds., Comprehensive Textbook of Psychiatry, Williams and Wilkins, Baltimore, 1995, pp. 899-902.

[39] B. S. Shastry, "Schizophrenia: A Genetic Perspective," International Journal of Molecular Medicine, Vol. 9, No. 3, 2002, pp. 207-212.

[40] S. Kapur and Y. Lecrubier, "Dopamine in the Pathophysiology and Treatment of Schizophrenia,” Martin Dunitz, London, 2003. http://dx.doi.org/10.3109/9780203427347

[41] M. F. Lenzenweger, G. McLachlan and D. B. Rubin, "Resolving the Latentstructure of Schizophrenia Endophenotypes Using Expectation-Maximizationbased Finitemixture Modeling,” Journal of Abnormal Psychology, Vol. 116, No. 1, 2007, pp. 16-29. http://dx.doi.org/10.1037/0021-843X.116.1.16

[42] S. Fisher and S. E. Cleveland, "Body Image and Personality,” Dover, New York, 1968. 
[43] A. Sims, "An Overview of the Psychopathology of Percaption: First Rank Symptoms as a Localizing Sign in Schizophrenia,” Psychopathology, Vol. 24, No. 6, 1991, pp. 369-374. http://dx.doi.org/10.1159/000284740

[44] B. Mitterauer, "The Loss of Self-Boundaries: Towards a Neuromolecular Theory of Schizophrenia,” Biosystems, Vol. 72, No. 3, 2003, pp. 209-215. http://dx.doi.org/10.1016/S0303-2647(03)00144-8

[45] American Psychiatric Association, "Diagnostic and Statistical Manual of Mental Disorders,” American Psychiatric Association, Washington, 1998.

[46] B. Mitterauer and W. F. Pritz, "The Concept of the Self: A Theory of Self-Observation," International Review in Psychoanalysis, Vol. 5, 1978, pp. 179-188.

[47] R. Arajärvi, T. Varilo, J. Haukka, J. Suvissari, J. Suokas, H. Juvonen, M. Muhonen, J. Lönnquist, et al., “Affective Flattening and Alogia Associate with Familial Form of Schizophrenia," Psychiatry Research, Vol. 141, No. 2, 2006, pp. 161-172.

http://dx.doi.org/10.1016/j.psychres.2005.08.008

[48] C. Holden, "Behavioral Genetics. Getting the Short End of the Allele," Science, Vol. 301, No. 5631, 2003, pp. 291-293. http://dx.doi.org/10.1126/science.301.5631.291a

[49] K. Hashimoto, G. Engberg, E. Shimizu, C. Nordin, L. H. Lindstrom and M. Iyo, "Elevated Glutamine/Glutamate Ratio in Cerebrospinal Fluid of First Episode and Drug Naive Schizophrenic Patients,” BMC Psychiatry, Vol. 5, 2005, p. 6. http://dx.doi.org/10.1186/1471-244X-5-6

[50] V. Faa, A. Cobana, F. Incani, L. Constantino, A. Cao and M. C. Rosatelli, "A Synchronous Mutation in the CFTR Gene Causes Aberrant Splicing in an Italian Patient Affected by a Mild Form of Cystic Fibrosis," Journal of Molecular Diagnostics, Vol. 12, No. 3, 2010, pp. 380-383. http://dx.doi.org/10.2353/jmoldx.2010.090126

[51] G. Wang and T. A. Cooper, "Splicing in Disease: Disruption of the Splicing Code and the Decoding Machinery," Nature Reviews Genetics, Vol. 8, No. 10, 2007, pp. 749761. http://dx.doi.org/10.1038/nrg2164

[52] L. Quintana-Murci, "Gene Losses in the Human Genome,” Science, Vol. 335, No. 6070, 2012, pp. 806-807. http://dx.doi.org/10.1126/science.1219299

[53] A. Poduri, G. Evrony, X. Cai and C. A. Walsh, "Somatic Mutation, Genomic Variation, and Neurological Disease," Science, Vol. 341, No. 6141, 2013, Article ID: 1237758.

[54] Z. Deng, J. L. Sobell and J. A. Knowles, "Epigenetic Alterations in Schizophrenia," Focus, Vol. 8, No. 3, 2010, pp. 358-365.

[55] B. A. Saakov, T. A. Khoruzhaya and E. A. Bardakhchyan, "Ultrastructural Mechanisms of Serotonin Demyelination," Bulleten Eksperimental noi Biologii I Meditsiny, Vol. 83, No. 5, 1977, pp. 606-610.

[56] E. Alberdi, M. V. Sanchez-Gomez, I. Torre, M. Domercq, A. Perez-Samatin, F. Pérez-Cerdá and C. Matute, "Activation of Kainate Receptors Sensitizes Oligodendrocytes to Complement Attack," Journal of Neuroscience, Vol. 26, No. 12, 2006, pp. 3220-3228. http://dx.doi.org/10.1523/JNEUROSCI.3780-05.2006
[57] L. R. Skelly, V. Calhoun, S. A. Meda, J. Kim, D. H. Mathalon and G. D. Pearlson, "Diffusion tensor Imaging in Schizophrenia: Relationship to Symptoms,” Schizophrenia Research, Vol. 98, No. 1, 2008, pp. 157-162. http://dx.doi.org/10.1016/j.schres.2007.10.009

[58] N. Takahashi, T. Sakurai, K. L. Davis and J. D. Buxbaum, "Linking Oligodendrocyte and Myelin Dysfunction to Neurocircuitry Abnormalities in Schizophrenia," Progress in Neurobiology, Vol. 93, No. 1, 2010, pp. 13-24. http://dx.doi.org/10.1016/j.pneurobio.2010.09.004

[59] M. N. Höstad, D. Segal, N. Takahashi, T. Sakurai, J. D. Buxbaum and P. R. Hof, "Linking White and Grey Matter in Schizophrenia: Oligodendrocyte and Neuron Pathology in the Prefrontal Cortex," Frontiers in Neuroanatomy, Vol. 3, 2009, p. 9. http://dx.doi.org/10.3389/neuro.05.009.2009

[60] R. D. Fields, “The Other Brain,” Simon and Schuster, New York, 2009.

[61] J. M. Robertson, “The Astrocentric Hypothesis: Proposed Role of Astrocytes in Consciousness and Memory Formation,” Journal of Physiology, Vol. 96, No. 3-4, 2002, pp. 251-255. http://dx.doi.org/10.1016/S0928-4257(02)00013-X

[62] B. Mitterauer, "Loss of Function of Glial Gap Junctions May Cause Severe Cognitive Impairments in Schizophrenia,” Medical Hypotheses, Vol. 73, No. 3, 2009, pp. 393-397. http://dx.doi.org/10.1016/j.mehy.2009.04.003

[63] R. C. Wolf, A. Höse, K. Frasch, H. Walter and N. Vasic, "Volumetric Abnormalities associated with Cognitive Deficits in Patients with Schizophrenia,” European Psychiatry, Vol. 23, No. 8, 2008, pp. 541-548. http://dx.doi.org/10.1016/j.eurpsy.2008.02.002

[64] C. D. Frith, "The Cognitive Neuropsychology of Schizophrenia,” Psychology Press, East Sussex, 1992.

[65] B. J. Mitterauer, "Qualitative Information Processing in Tripartite Synapses: A Hypothetical Model,” Cognitive Computation, Vol. 4, No. 2, 2011, pp. 181-194. http://dx.doi.org/10.1007/s12559-011-9115-2

[66] B. Mitterauer, "Nonfunctional Glial Proteins in Tripartite Synapses: A Pathophysiological Model of Schizophrenia," Neuroscientist, Vol. 11, No. 3, 2005, pp. 192-198. http://dx.doi.org/10.1177/1073858404265745

[67] N. Markis, L. J. Seidman, T. Ahern, D. N. Kennedy, V. S. Caviness, M. T. Tsuang and J. M. Goldstein, "White Matter Volume Abnormalities and Association with Symptomatology in Schizophrenia,” Psychiatry Research, Vol. 183, No. 1, 2010, pp. 21-29. http://dx.doi.org/10.1016/j.pscychresns.2010.04.016

[68] M. V. Sofroniew, "Reactive Astrocytes in Neural Repair and Protection,” Neuroscientist, Vol. 11, No. 5, 2005, pp. 400-407. http://dx.doi.org/10.1177/1073858405278321

[69] A. S. Brown, "The Risk for Schizophrenia from Childhood and Adulthood Infections," American Journal of Psychiatry, Vol. 165, No. 1, 2008, pp. 7-10. http://dx.doi.org/10.1176/appi.ajp.2007.07101637 